\title{
EDITORIAL
}

\section{PIE DIABÉTICO: UNA SERIA COMPLICACIÓN DE LA DIABETES}

\author{
DIABETIC FOOT: A SERIOUS COMPLICATION OF DIABETES
}

La diabetes mellitus (DM) es un importante problema de salud pública con una prevalencia creciente en todo el mundo. Se estima que aumente a 642 millones de la población en 20401. Probablemente el crecimiento de la prevalencia de la DM se acompañe de un incremento de sus complicaciones y que éstas provoquen un agravamiento de la morbilidad, la mortalidad y el gasto en salud debido a la necesidad de atención especializada.

El pie diabético es una de las complicaciones más relevantes y devastadoras de la DM, y se define como un grupo de síndromes en los cuales la neuropatía, la isquemia y la infección conducen a la ruptura del tejido y la posible amputación; y aunque son prevenibles, representan una de las causas más frecuentes de ingreso hospitalario en los países occidentales.

En 2005 la Federación Internacional de Diabetes (FID), dada la importancia de la enfermedad de pie diabético, decidió crear conciencia con una campaña mundial para "poner los pies primero" y resaltar el problema común de amputación entre los pacientes con DM.

El desarrollo de una úlcera es un evento fundamental en la vida de un paciente con DM, además de un marcador de enfermedad grave y comorbilidades. La incidencia de úlceras se ubica entre el 4 y el $10 \%$. Estos pacientes presentan un mayor riesgo de muerte prematura, infarto de miocardio y accidente cerebrovascular fatal. El riesgo de amputación de extremidades inferiores es de 15 a 40 veces mayor en personas con DM que en personas sin DM. Cada 20 segundos se pierde una extremidad inferior por DM en el mundo y es la causa más común de amputación no traumática de extremidades inferiores ${ }^{2}$. El riesgo de vida de un paciente con DM que desarrolla una úlcera en el pie se estima en un $25 \%$.

Numerosos pacientes en el momento del diagnóstico de DM2 tienen una neuropatía significati- va. En el estudio prospectivo de diabetes del Reino Unido, el $13 \%$ de los pacientes en el momento del diagnóstico tenía una neuropatía lo suficientemente grave como para establecerse en riesgo de ulceración del pie.

La neuropatía periférica aumenta el riesgo de desarrollo de úlceras a través de la pérdida de las sensaciones protectoras y las deformidades del pie; esto se asocia con un importante incremento de la posibilidad de ulceración.

De ahí que Paul Brand haya documentado que "el dolor es el regalo más grande de Dios para la humanidad" porque la pérdida del dolor y de la sensación acrecienta las posibilidades de desarrollar úlceras en los pies. A pesar del daño tisular y de la infección, los pacientes continúan caminando lo que complica aún más la situación.

Los pacientes diabéticos con úlceras en los pies se encuentran con el estigma, la pérdida del rol social, el aislamiento social y el desempleo.

La úlcera del pie es una enfermedad costosa y debilitante con graves consecuencias. Además la mortalidad después de las amputaciones de las extremidades inferiores en pacientes con DM varía del 39 al $80 \%$ a los cinco años. Más de la mitad de todas las amputaciones no traumáticas de extremidades inferiores se debe a la DM.

La amputación de la extremidad causa distorsión de la imagen corporal, aumento de la dependencia, pérdida de la productividad e incremento de los costos del tratamiento de las úlceras del pie.

Respecto de los costos, hace aproximadamente 10 años, Rogers et al. informaron que se gastaron 18 mil millones de dólares en la atención de las úlceras de pie y 11,7 mil millones de la misma moneda en amputaciones de extremidades inferiores. Recientemente datos del Reino Unido sugieren que una estimación conservadora del costo anual de los problemas de pie diabético supera los 600 millones de libras esterlinas ${ }^{3}$, lo que represen- 
ta aproximadamente el $1 \%$ del presupuesto total del Servicio Nacional de Salud.

Estos estudios apenas consideran el costo para el sistema de salud que es el costo directo. Los costos indirectos se estiman entre el $40 \mathrm{y}$ $50 \%$ del total para una patología crónica. Pero las consecuencias en cuanto a la calidad de vida de los pacientes tampoco son consideradas.

En la práctica clínica la mayoría de los médicos no examina los pies de los pacientes con DM adecuadamente y tampoco brinda educación con respecto al cuidado de los pies. Existe entonces una urgente necesidad de estrategias preventivas para reducir la incidencia de complicaciones del pie entre las personas con DM. En este sentido, realizar trabajos de investigación para obtener datos acerca de la prevalencia de DM y de la patología del pie en

\section{BIBLIOGRAFÍA}

1. IDF. Diabetes Atlas. International Diabetes Federation, Brussels. 2015. Disponible en: https://www.idf.org/elibrary/epidemiology-research/diabetes-atlas.html (acceso 10 de septiembre de 2017). nuestro país como el presentado por Gabriela V. Carro y col., ayuda a la implementación de programas con el fin de prevenir esta patología.

La educación del paciente y de la familia es fundamental, como así también el entrenamiento del equipo profesional para disminuir los gravísimos problemas sociales y económicos que esta patología produce. El examen y cuidado periódico de los pies de las personas con DM deben incluirse dentro de los requisitos de evaluación obligatoria en la confección de la historia clínica, al igual que los controles de la presión arterial y la glucemia.

\section{Dr. Ricardo Antonucci Médico de la División Nutrición Hospital de Clínicas UBA}

2. The International Working Group on the Diabetic Foot 2017. Disponible en: http://iwgdf.org/ (acceso 10 de septiembre de 2017).

3. Kerr M, Rayman G, Jeffcoate WJ. Cost of diabetic foot disease to the National Health Service in England. Diabetic Medicine 2014 Dec 1;31(12):1498-504. 